\title{
Distinguishing Commercial Beers Using a Solution-Based Sensor Array Derived from Nanoscale Polydiacetylene Vesicles
}

\author{
Qian, Xiaomin; Gargalo, Carina L.; Gernaey, Krist V.; Stadler, Brigitte
}

Published in:

ACS Applied Nano Materials

Link to article, DOI:

10.1021/acsanm.0c00187

Publication date:

2020

Document Version

Publisher's PDF, also known as Version of record

Link back to DTU Orbit

Citation (APA):

Qian, X., Gargalo, C. L., Gernaey, K. V., \& Stadler, B. (2020). Distinguishing Commercial Beers Using a Solution-Based Sensor Array Derived from Nanoscale Polydiacetylene Vesicles. ACS Applied Nano Materials , 3(4), 3439-3448. https://doi.org/10.1021/acsanm.0c00187

\section{General rights}

Copyright and moral rights for the publications made accessible in the public portal are retained by the authors and/or other copyright owners and it is a condition of accessing publications that users recognise and abide by the legal requirements associated with these rights.

- Users may download and print one copy of any publication from the public portal for the purpose of private study or research.

- You may not further distribute the material or use it for any profit-making activity or commercial gain

- You may freely distribute the URL identifying the publication in the public portal 


\title{
Distinguishing Commercial Beers Using a Solution-Based Sensor Array Derived from Nanoscale Polydiacetylene Vesicles
}

\author{
Xiaomin Qian, Carina L. Gargalo, Krist V. Gernaey, and Brigitte Städler* \\ Cite This: https://dx.doi.org/10.1021/acsanm.0c00187 \\ Read Online
}

ABSTRACT: The fast and effective discrimination of different liquid mixtures such as beers is a long-standing challenge in the food and beverage industry. We report the fabrication of a solution-based sensor array derived from nanoscale polydiacetylene (PDA) vesicles to distinguish different ethanol containing aqueous solutions supplemented with different model flavor compounds relevant in beers. The sensitivity of the sensors was significantly affected by the head group modification of the diacetylenes and the composition of the sensors. Finally, a selected set of sensors was

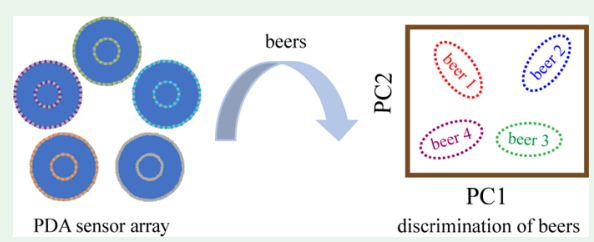
utilized as a sensor array to distinguish different commercial beers in combination with statistical data analysis. This approach represents an effort toward the employment of low-cost sensors with an easy read-out for the fast discrimination of different beverages.

KEYWORDS: diacetylene, polydiacetylene, self-assembly, sensor array, nanoscale vesicles, beer discrimination, principal component analysis, hierarchical clustering analysis

\section{INTRODUCTION}

Methods for the fast and reliable discrimination of complex mixtures, such as dairy products and alcoholic/nonalcoholic beverages, are of interest for product development, quality, and safety control in the food industry. Due to the great complexity of flavors and odors in beverages, as well as their subjective nature, the current predominant analytical approaches remain rather complex and labor-intense, focusing on gas chromatography coupled with mass spectrometry (GC-MS) and sensory panels. ${ }^{1,2}$ Electronic tongues, ${ }^{3}$ employing potentiometric/ amperometric chemical sensors and electrochemical techniques, are an emerging concept but many technical, material, and computational challenges need to be tackled before they can become widely applicable. Alternative concepts, which would allow for the fast on-site screening, are therefore in high demand.

Inspired by the fact that in nature, sensing taste and smell is usually accomplished through the cooperation of a group of nonspecific receptors, sensor arrays are becoming more and more popular. Unlike the traditional specific lock-and-key sensors, which are usually highly sensitive to a specific analyte, the sensors in an array have different selectivity and sensitivity toward different analytes. Each sensor used in the array provides the chemical dimensionality to the array data, and distinct response patterns are obtained for each analyte. The differentiation between different analytes is achieved via a pattern-recognition data analysis process. Such differential sensing is especially suitable for the analysis of complex mixtures, which are often difficult to separate using a singlecomponent chemical sensor. The most important step in constructing a chemical sensor array is to design and synthesize the different chemical sensors that determine the discriminative ability of the array. Especially interesting in this context are colorimetric/fluorescent sensors for instance based on polydiacetylenes (PDAs) as recently reviewed by $\mathrm{us}^{4}$ and others. $^{5-7}$ PDAs are a class of conjugated polymers that have been extensively exploited since the first report in $1969 .^{8}$ Diacetylene (DA) monomers can be polymerized into PDAs with UV light irradiation via a 1,4-addition reaction, resulting in a typically blue colored polymer within a few minutes without the need of a catalyst, an initiator, or heat. Depending on the chemical structures of the DA monomers, PDAs can be tailor-made to be responsive to various external stimuli including temperature, ${ }^{9-15} \mathrm{pH}^{16,17}$ light, ${ }^{18}$ mechanical stress, ${ }^{19,20}$ microorganisms, ${ }^{21,22}$ biomolecules $^{23,24}$ and so forth, usually accompanied by a blue-to-red (and nonfluorescent to fluorescent) color shift, which is easily detectable using a spectrometer or even by the naked eye. PDA sensors can be constructed in various forms, including as films on various supports, ${ }^{25,26}$ as nanoparticles in aqueous solution, ${ }^{27-29}$ or embedded into solid matrices, ${ }^{30-32}$ depending on the chemophysical features of the DA monomers and the environment in which the PDAs are intended to be used. Among them, nanoparticles prepared by the self-assembly of amphiphilic DAs in aqueous solution are the most commonly

Received: January 22, 2020

Accepted: March 12, 2020 
Scheme 1. Development of a Solution-Based Sensor Array Derived from Nanoscale PDA Vesicles ${ }^{a}$

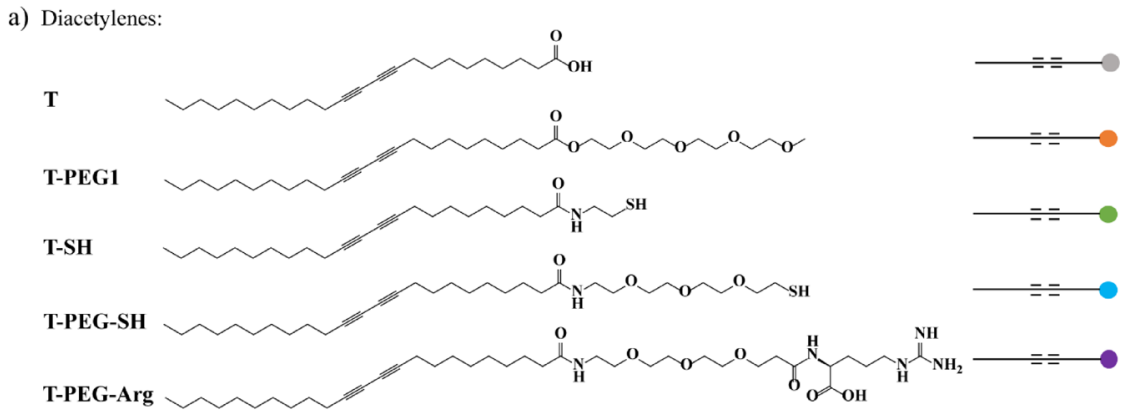

b) Sensor array:

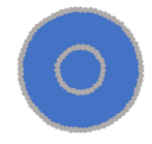

sensor $\mathbf{T}$

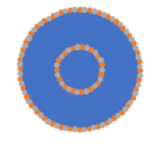

sensor $\mathbf{T}+\mathbf{T}$-PEG1

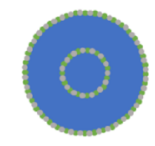

sensor $\mathbf{T}+\mathbf{T}-\mathbf{S H}$

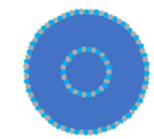

sensor T+T-PEG-SH sensor T+T-PEG-Arg

c) Sensing concept:

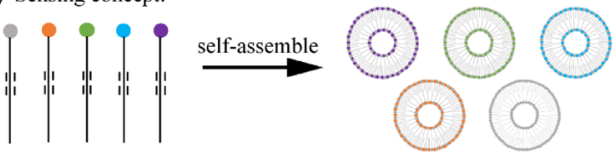

Diacetylenes with different head groups

Self-assembled diacetylene vesicles

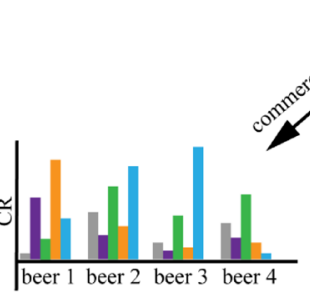

Colorimetric responses of different beers in different sensors

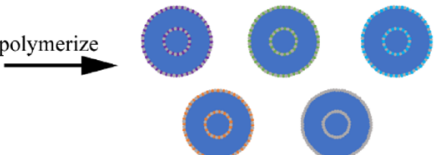

Blue PDA vesicular sensors

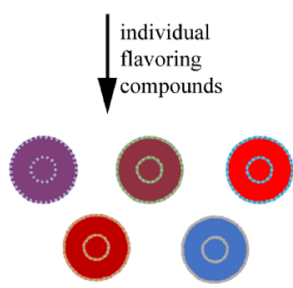

Color change of different sensors toward individual flavoring compounds

${ }^{a}$ (a) Five DA monomers with different head groups used to assemble the nanoscale vesicular PDA sensors, including a carboxylic acid group (T), a short PEG chain (T-PEG1), a thiol group (T-SH), a PEGylated thiol group (T-PEG-SH), and a PEGylated arginine (T-PEG-Arg). (b) Five vesicular PDA sensors contained in the sensor array. (c) Schematic illustration of the discrimination of beers using the solution-based PDA sensor array: grouping of the beers using principal component analysis (PCA) on the basis of the different colorimetric responses of the sensors to different beers.

explored form of PDAs for sensing. In general, such solutionbased sensors have several advantages over other forms, such as easy preparation using hydration methods, fast read-out using multiwell plate readers, and biomimicry due to their lipid membrane-like appearance, and they were used to target various analytes such as surfactants, ${ }^{33,34}$ bacteria, ${ }^{35,36}$ metal ions, ${ }^{37}$ etc. Although various applications for solution-based PDA sensors were considered, their usage in the context of food and beverage safety, product development, and process monitoring remains largely unexplored, and mostly limited to sanitizer and surfactants, ${ }^{38}$ as well as bacteria detection in food samples. $^{21,35,39-41}$

Herein, we report the development of a solution-based sensor array derived from nanoscale PDA vesicles to discriminate commercial beers including advanced statistical analysis in an effort to combine low-cost sensors with fast readout possibility. Specifically, we (i) synthesized various DA monomers with analyte-targeting head groups from a commercial DA 10,12-tricosadiynoic acid (Scheme 1a), (ii) used these different DAs to assemble nanosized vesicular PDA sensors, and employed them (iii) to detect EtOH and two specific flavoring molecules (4-vinyl guaiacol and diacetyl) and (iv) to discriminate commercial beers (Scheme 1b,c) in combination with statistical data analysis.

\section{EXPERIMENTAL SECTION}

Materials and Instruments. All the chemicals were purchased from commercial suppliers (Sigma-Aldrich, Alfa Aesar, Acros Organics, and VWR) and used without further purification unless specified. Commercial DAs, 10,12-tricosadiynoic acid (T), 10,12pentacosadiynoic acid (P), and 5,7-hexadecadiynoic acid $(\mathbf{H})$ were purified by first dissolving in chloroform and then filtered through a filter paper to remove the insoluble polymer parts. Then, the filtrate was dried using a rotary evaporator with the flask protected by aluminum foil. The obtained white powder was used to prepare vesicles or immediately for the synthesis of other DAs. Ultrapure water was obtained from an ELGA Purelab Ultra system (ELGA LabWater, Lane End). 4-Vinyl guaiacol (4-VG) was purified with column chromatography before use to remove the impurities. Thin layer chromatography (TLC) plates were used to monitor the reactions. Visualization of the developed TLC plates was done under UV light (254 and $365 \mathrm{~nm})$. Silica gel (250-430 mesh) was used as stuffing in the glass column for column chromatography. ${ }^{1} \mathrm{H}-\mathrm{NMR}$ and ${ }^{13} \mathrm{C}-\mathrm{NMR}$ spectra were recorded on a Bruker Ascend $400 \mathrm{NMR}$ spectrometer. Chemical shifts are given in parts per million (ppm) with tetramethylsilane (TMS) as the reference. Mass spectra (MS) were recorded on a Waters GC-TOF and LC/Q-TOF mass 
spectrometer. As for the characterization of the compounds synthesized, for the compounds that had been reported previously, only ${ }^{1} \mathrm{H}-\mathrm{NMR}$ was conducted and compared with the literature to ensure the purity and structural correctness. For all the new compounds, ${ }^{1} \mathrm{H}-\mathrm{NMR},{ }^{13} \mathrm{C}-\mathrm{NMR}$, and HRMS were conducted to confirm the structure. Polymerization of the DAs was conducted with a hand-held UVP UVLS-26 EL Series UV lamp (254 nm, 6 watts). Dynamic light scattering (DLS) was conducted with a Malvern Zetasizer Nano 590 to determine the hydrodynamic radius of the vesicles ( $\mathrm{Z}$ average size). $\mathrm{UV}$-vis absorption spectra were all taken on a PerkinElmer EnSight multimode plate reader with either 48-well plates or 96-well plates. Tip sonication was conducted on a Branson digital sonifier model 450 with a $20 \%$ amplitude. GraphPad Prism 8 was used to process the data to remove outliers and to determine statistically significant differences. Principal component analysis and hierarchical clustering analysis were performed using RStudio and the MATLAB PLS Toolbox, respectively.

Synthetic Procedures. Detailed synthetic procedures and characterization data of the compounds can be found in the Supporting Information.

Preparation of PDA Sensors. PDA sensors were prepared via a typical hydration method, as illustrated in Scheme S2. For a singlecomponent sensor, $0.01 \mathrm{mmol} \mathrm{DA}$ monomer was weighed into a glass vial and dissolved in $1 \mathrm{~mL}$ of chloroform to reach a concentration of $10 \mathrm{mM}$. Then, $200 \mu \mathrm{L}$ of the solution was transferred into a roundbottom flask. For a two-component sensor, each DA monomer (0.01 $\mathrm{mmol}$ ) was first dissolved in $1 \mathrm{~mL}$ of chloroform. Then, $100 \mu \mathrm{L}$ of each solution was transferred into a round-bottom flask to mix it well. Chloroform was removed by gently blowing dry with a $\mathrm{N}_{2}$ stream, and a thin film was obtained on the wall of the flask. Then, $10 \mathrm{~mL}$ of ultrapure $\mathrm{H}_{2} \mathrm{O}$ was added into the flask to reach a concentration of 0.2 $\mathrm{mM}$ and it was subjected to tip sonication for $30 \mathrm{~min}$. After that, the obtained milky solution was filtered through a $0.8 \mu \mathrm{M}$ cellulose acetate membrane to remove large aggregates. The solution was then transferred into a glass vial and kept in the fridge $\left(4{ }^{\circ} \mathrm{C}\right)$ overnight. Polymerization was then carried out by irradiating the solution sample with the hand-held UV lamp for $2 \mathrm{~min}$. The distance from the lamp to the sample was kept constant at $3 \mathrm{~cm}$ throughout the whole preparation process for all the sensors. The appearance of a blue color indicated that the polymerization of the DA monomers occurred. The obtained blue solution was subsequently used for sensing experiments. Sensors prepared from a single component will be referred to as sensor A from now on in which A represents the diacetylene used to prepare the sensors. Sensors made from two diacetylenes will be referred to as sensor $\mathrm{A}+\mathrm{B}$ in which $\mathrm{A}$ and $\mathrm{B}$ represent the two DAs used to prepare the sensors, respectively.

Sensing Experiments. Detection of EtOH. Sensor solution (300 $\mu \mathrm{L}$ ) was transferred into four wells (48-well plate). From left to right, $300,276,252$, and $228 \mu \mathrm{L}$ of water were added into the four wells. Then, from left to right, $0,24,48$, and $72 \mu \mathrm{L}$ of EtOH were added to reach a total volume of $600 \mu \mathrm{L}$ in each well. Subsequently, the absorbance was scanned from 400 to $700 \mathrm{~nm}$ using a multiplate reader. These experiments were performed with at least five independent repeats.

Detection of Flavoring Molecules. Sensor solution $(300 \mu \mathrm{L})$ was transferred into five wells (48-well plate). From left to right, 300, 297, 270,150 , and $0 \mu \mathrm{L}$ of water were added into the five wells. Then, 0,3 , 30,150 , and $300 \mu \mathrm{L}$ of analyte solution ( $2 \mathrm{mM}$ in water) were added to reach a total volume of $600 \mu \mathrm{L}$ in each well. The mixtures were shaken well and then subjected to a multiplate reader to measure the absorbance. These experiments were performed with at least five independent repeats.

Detection of Beer Samples. Sensor solution $(300 \mu \mathrm{L})$ was transferred into eight wells (48-well plate). Then, $300 \mu \mathrm{L}$ of water was added into each well and the absorbance was measured with water as the baseline. Then, $300 \mu \mathrm{L}$ of sensor solution was transferred into eight wells from left to right in a new row. After that, $300 \mu \mathrm{L}$ of the beer was added and absorbance was measured with the mixture of water $(300 \mu \mathrm{L})$ and beer $(300 \mu \mathrm{L})$ as the baseline. As different beers show different colors intrinsically, it is crucial to use a solution without the sensors, namely, a mixture consisting of equivalent volumes of water and beer, as the baseline to account for the interference of the background colors of the beers.

Evaluation of Sensitivity. When being used as colorimetric sensors, the sensitivity of PDAs toward certain analytes is calculated based on absorbance and is quantitatively evaluated by the colorimetric response (CR), which is defined as the relative change from blue to red. ${ }^{42} \mathrm{CR}$ is calculated as

$$
\mathrm{CR}=\frac{\left(\mathrm{PB}_{\mathrm{b}}-\mathrm{PB}_{\mathrm{a}}\right)}{\mathrm{PB}_{\mathrm{b}}} \times 100 \%
$$

where $\mathrm{PB}_{\mathrm{b}}$ and $\mathrm{PB}_{\mathrm{a}}$ are the before and after percentages of blue (PB) of the system. Meanwhile, $\mathrm{PB}$ is defined as

$$
\mathrm{PB}=\frac{A_{\text {blue }}}{A_{\text {blue }}+A_{\text {red }}} \times 100 \%
$$

where $A_{\text {blue }}$ and $A_{\text {red }}$ are the absorbance of the blue form $(\lambda=\sim 650$ $\mathrm{nm})$ and the red form $(\lambda=\sim 550 \mathrm{~nm})$ of the system, respectively. Therefore, a higher CR indicates a larger color change from blue to red of the system, which means the sensor is more sensitive to that analyte.

It is worth mentioning that a purple color was also frequently observed during the preparative and sensing process of the sensors, which did not affect the calculation of CRs as the two absorption peaks are always at $\lambda \approx 650$ and $\lambda \approx 550 \mathrm{~nm}$. The color of the solution was dependent on the ratios of the absorbance at these two wavelengths.

\section{RESULTS AND DISCUSSION}

Influence of Side Chains. It is well known that the chemical structures, namely, the side chains and the head groups of DAs significantly affect the sensitivity and selectivity of PDAs when being used as sensors. In order to investigate the influences of the integral topological structure of the side chains on the stability of PDAs, three commercially available DAs, $\mathbf{T}, \mathbf{P}$, and $\mathbf{H}$, and three analogues with different side-chain lengths (6,8-nonadecadiynoic acid, 4,6-heptadecadiynoic acid, and 12,14-pentacosadiynoic acid) synthesized using the traditional Cadiot-Chodkiewicz coupling reaction, ${ }^{43}$ were compared. Even though all the six DAs could be polymerized into blue PDAs at the solid state with UV light irradiation, differences were observed in the solution (Supporting Information, Table S1). Sensor $\mathbf{H}$ with a shorter alkyl chain was responsive to light as the blue solution turned purple after $24 \mathrm{~h}$ and further reddish after $48 \mathrm{~h}$ while being stored at room temperature (Supporting Information, Figure S1), which is in accordance with the previous result that shortening the alkyl chains of diacetylene leads to faster response to external stimuli. ${ }^{44}$ The vesicles prepared from DAs 6,8-nonadecadiynoic acid, 4,6-heptadecadiynoic acid, and 12,14-pentacosadiynoic acid were unstable, likely due to the high hydrophobicity. Precipitates were constantly formed during the self-assembly process, even when the concentrations were as low as 0.02 $\mathrm{mM}$. The vesicles of $\mathbf{T}$ and $\mathbf{P}$ before and after polymerization were considered stable. A blue color was observed upon exposure to UV light within a few seconds, which could be used as a quick "benchtop" test of vesicle formation. The blue color sustained and no precipitations were observed after $48 \mathrm{~h}$. When comparing the sensitivity of sensors $\mathbf{T}$ and $\mathbf{P}$ to different amounts of $\mathrm{EtOH}$ in water, no significant differences were observed (Supporting Information, Figure S2). T was chosen for the following experiments and all the other DA derivatives with modified head groups were synthesized from $\mathbf{T}$.

Hydrophilicity of PDA Sensors. In the case of PDA vesicle formation, the DA monomers must hydrate and self- 
assemble into the optimal packing and orientation to undergo the 1,4-addition to form PDAs. Therefore, two factors have to be taken into consideration when designing new DA monomers, namely, the amphiphilicity of the monomers and the noncovalent interactions between the head groups of the monomers. The former promotes the formation of a bilayer vesicular structure, while the latter stabilizes the vesicles. A poly(ethylene glycol) (PEG) chain was introduced as a way to improve the amphiphilicity of the DAs. Three DAs with different lengths of PEG chains (T-PEGx) as head groups, TPEG1 (208 Da PEG), T-PEG2 (550 Da PEG), and T-PEG3 (5000 Da PEG) were synthesized to investigate the influence of the PEG chain length on the vesicular PDA sensor sensitivity. The polymerization of vesicles assembled from pure T-PEGx did not occur, possibly due to the weak intermolecular interaction between the monomer head groups, which resulted in poor topological packing of the molecules. Therefore, the vesicles were assembled using T-PEGx and T to determine the sensitivity of the PDA sensors toward EtOH. The hydrodynamic size distribution of the vesicles was assessed using dynamic light scattering (DLS). The results suggested that the incorporation of a second DA monomer had a significant influence on the size distribution of the vesicles while polymerization had a negligible effect (Supporting Information, Table S2). The significant size increase after incorporation of T-PEGx was due to the presence of the PEG chains that substantially increased the hydrodynamic radius, which was the most pronounced for the highest molecular weight PEG.

Assembly. Previously, it has been reported that the irradiation dose has a significant influence on the stability of PDA vesicles in solution. ${ }^{45}$ Namely, increasing the irradiation time enhanced the susceptibility of the vesicles to red-phase conversion, indicating the metastability of the blue form. In turn, the stability of the vesicles affects their sensitivity. This phenomenon usually happens when the vesicles consist of more than one component, such as two different DAs or DAs mixed with lipids. We first examined the stability of the vesicles formed from pure $\mathbf{T}$ to identify the optimal UV irradiation time. The results indicated that for sensor $T$, prolonging the irradiation time only led to an enhancement of absorbance (Supporting Information, Figure S3a), which reached a maximum after $12 \mathrm{~min}$. While the percentage of blue (PB) of the system remained almost unchanged during the whole irradiation process even with an irradiation time of up to 60 min (Supporting Information, Figure S3b). The blue color was retained during the whole process, suggesting that sensor $\mathbf{T}$ was stable and not affected by the UV dose. On the other hand, sensor T+T-PEG1 turned from blue to red with increasing irradiation time ( $t=0$ to $40 \mathrm{~min}$ ) as the absorption peak at $650 \mathrm{~nm}$ gradually decreased, while the absorption peak at $548 \mathrm{~nm}$ gradually enhanced (Figure 1a). The PB of the system decreased from 70 to $10 \%$ after $40 \mathrm{~min}$ (Supporting Information, Figure S4a), and the solution became red in the end (Supporting Information, Figure S4b). This result indicated that the incorporation of T-PEG1 labilized the system as compared to PDA sensors made from pure T. To determine the optimal irradiation time, sensitivity of sensor $\mathbf{T}$ +T-PEG1 toward $8 \mathrm{vol} \% \mathrm{EtOH}$ in water was measured as a function of irradiation time. It was found that the sensor showed the highest $\mathrm{CR}$ at an irradiation time of $2 \mathrm{~min}$ (Figure 1b). After $10 \mathrm{~min}$, although the PB of the system kept decreasing as the irradiation time was prolonged, the $\mathrm{CR}$
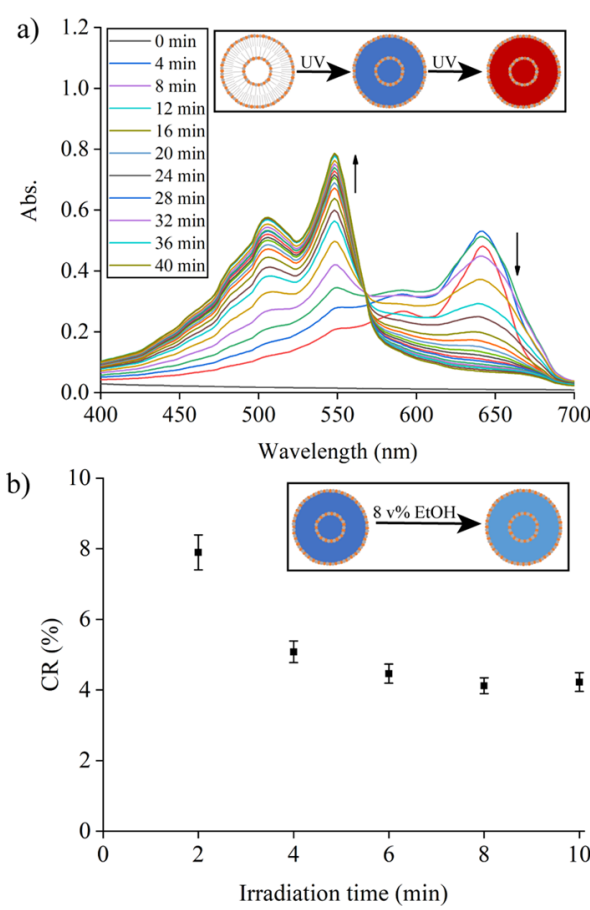

Figure 1. (a) Time-dependent UV-vis spectra $(0-40 \mathrm{~min})$ of sensor T+T-PEG1 upon irradiation with UV light. The arrows indicate the variation trend of the two absorption peaks. (b) Colorimetric responses of sensor T+T-PEG1 toward $8 \mathrm{vol} \% \mathrm{EtOH}$ in water at different irradiation times. The data are expressed as mean $\pm \mathrm{SD}$. $(n=$ $5)$.

remained stable. Therefore, we chose an irradiation time $t=2$ min at which point the PDA sensor made from T+T-PEG1 showed the highest $\mathrm{PB}$ and highest sensitivity to 8 vol \% EtOH.

EtOH Detection. Considering the fact that $\mathrm{EtOH}$ is the second most dominating compound after water in alcoholic beverages such as beers, understanding the influence of $\mathrm{EtOH}$ concentration on the sensitivity of the sensors is important. It is not surprising that sensor $\mathbf{T}$ was responsive to $\mathrm{EtOH}$ as solvatochromism is one of the most well-known features of PDAs. ${ }^{46-48}$ The main absorption peak of sensor $\mathbf{T}$ clearly blueshifted with an increasing amount of EtOH, accompanied by an increase in $\mathrm{CR}$ and a distinct color change (Supporting Information, Figure S5). When the EtOH content was above 70 vol \%, the system became saturated and the CR reached a plateau. However, because most beers have an alcohol content below 12 vol \%, sensor $\mathrm{T}$ exhibited limited sensitivity at low EtOH concentrations.

Therefore, DAs with PEGylated head groups were considered for the assembly of the sensors to improve the sensitivity of sensor $\mathbf{T}$ to low EtOH concentrations for two main reasons. First, PEG chains improve the amphiphilicity of the DA monomers, which is vital for the formation of vesicular structure and prevents DAs from precipitating in water. Second, the incorporation of a second component to the bilayer vesicular structure enhances the fluidity of the system. Sensors with a higher fluidity are thought to behave more sensitively to external perturbations. Therefore, the sensitivity of the PDA sensors to EtOH was first evaluated focusing on the influence of the PEG chain length. Considering that the molar ratio between the two DAs used for assembly affects the sensitivity of PDA sensors, as shown in previous reports, ${ }^{49,50}$ 
the molar percentage of $\mathbf{T}$ was varied from 40 to $100 \%$. When the molar percentage of $\mathbf{T}$ was below $40 \%$, the system did not polymerize after UV irradiation for $2 \mathrm{~min}$ as the solution did not show the expected blue color but remained colorless. This observation suggested the failure of the self-assembly process into vesicular structures in the solution with a too low amount of $\mathbf{T}$ because of the weak intermolecular interactions among the head groups of DAs. Constant 12 vol \% EtOH was chosen as the analyte solution (Figure $2 \mathrm{a}$ ). First, the incorporation of
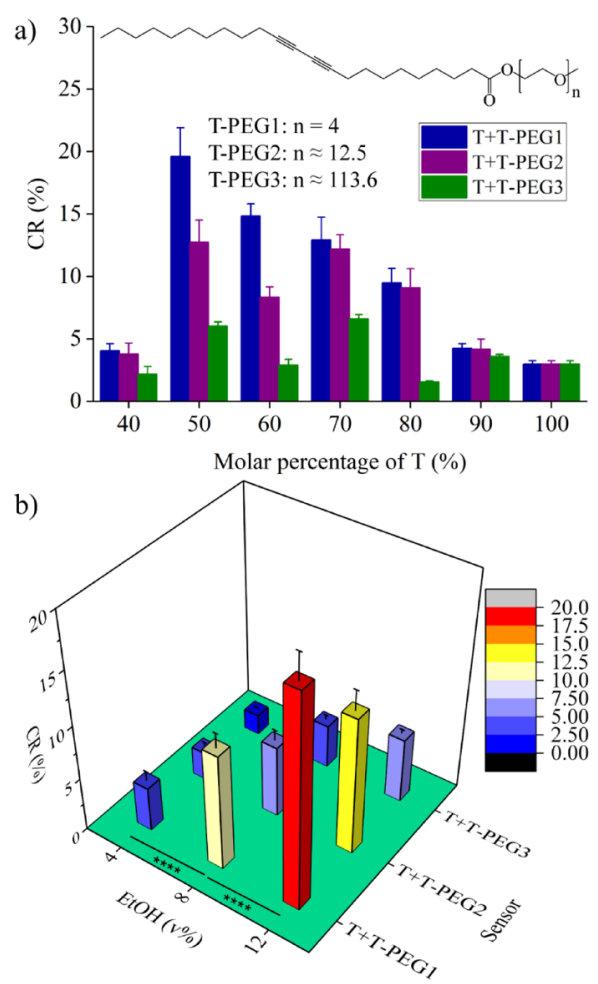

Figure 2. EtOH detection: (a) colorimetric responses of sensor $\mathbf{T}+\mathbf{T}$ PEG1, sensor T+T-PEG2, and sensor T+T-PEG3 toward $12 \mathrm{vol} \%$ of EtOH in water. (b) Colorimetric responses of sensor T+T-PEG1, sensor T+T-PEG2, and sensor T+T-PEG3 (molar percentage of $\mathbf{T}$ was $50 \%$ ) toward different concentrations of $\mathrm{EtOH}$ in water. The data are expressed as mean $\pm \mathrm{SD}$. $(n=5, * * * * p<0.0001)$.

any of the three T-PEGx enhanced the sensitivity of the sensors to EtOH, as compared to sensor T. Sensor $\mathbf{T}$ had almost no sensitivity to $12 \mathrm{vol} \% \mathrm{EtOH}$ with a low CR ( 3\%), while the majority of the T-PEGx containing sensors showed higher $\mathrm{CR}$, suggesting that the hydrophilicity of the monomer improved the sensitivity to EtOH. Second, the molar ratio of the two DAs played a vital role in affecting the sensitivity. Taking sensor T+T-PEG1 as an example, while $90 \%$ T showed a CR of $\sim 4 \%$, showed a significantly higher CR of $\sim 19 \%$. A similar phenomenon was also observed for sensor T+T-PEG2 and sensor T+T-PEG3. The highest sensitivity toward 12 vol $\% \mathrm{EtOH}$ was observed when the sensor consisted of $50 \% \mathrm{~T}$ and 50\% T-PEG1, namely, when the molar ratio between the two monomers was $1: 1$. It is worth mentioning that when the sensors consisted of $40 \% \mathrm{~T}$, the responses were quite low as compared to the adjacent sensors consisting of 50\% $\mathrm{T}$. This was probably due to the inadequate self-assembly of the two monomers, which could also be seen from the very light blue color of the sensors. Similar trends were seen for sensor $\mathbf{T}+\mathbf{T}$ PEG1 when the volumetric content of $\mathrm{EtOH}$ was varied
(Supporting Information, Figure S6). Further, the length of the PEG chain also significantly affected the sensitivity of the sensors. For the three sensors composed of the same molar amount of $\mathbf{T}$, a sensor with a shorter PEG chain always showed higher sensitivity as compared to a sensor with a longer PEG chain. This influence was clearly more significant when the sensors contained higher molar amounts of monomer T-PEGx. The PEG chain length-dependent sensitivity to EtOH was probably due to the nature of PEG (Supporting Information, Scheme S3). When the PEG chains were short, EtOH molecules could interact freely with the carboxylic acid groups of $\mathbf{T}$, which rendered the distortion of the polymer backbone and thus led to the color change of the system. However, when the PEG chains were long enough, the vesicles were wrapped around by the PEG chains. The cross-linking entanglement of the chains made the vesicles less exposed to EtOH molecules, thus leading to a lower CR. Furthermore, depending on the molecular weight of the PEG chains, the stronger hydrogen bonding interactions between the long PEG chain and water molecules also provided a stronger shielding effect for the vesicles, probably leading to lower sensitivity toward EtOH. Since sensor T+T-PEG1 showed much higher CRs than sensor $\mathbf{T}$ and sensor T+T-PEG2 to EtOH, we could assume that the chosen PEG chain length was likely close to the optimum. Further, we would like to note that the simple blending of PEG chains with $\mathbf{T}$ would likely not give the same results as the PEG chains would dissolve in water and not form vesicles together with $\mathbf{T}$.

Considering the fact that most beers have an EtOH content below $12 \mathrm{vol} \%$, it is important to see if the sensors still work at lower EtOH concentrations. Gradient concentrations of EtOH in water $(4,8$, and 12 vol\%) were tested with the three $\mathbf{T}+\mathbf{T}$ PEGx sensors with a fixed $50 \%$ molar content of $\mathbf{T}$ since this ratio showed the best result with 12 vol \% EtOH. First, with an increasing amount of $\mathrm{EtOH}$, increasing CRs were observed for all the three sensors (Figure $2 \mathrm{~b}$ ). T+T-PEG1 with the shortest PEG chain always showed the highest CRs as compared to $T$ +T-PEG2 and T+T-PEG3 at different EtOH concentrations, which was in accordance with the results obtained above. For sensor T+T-PEG1, significant differences were observed among different EtOH concentrations, indicating the good sensing ability of the sensor at low concentrations. Based on the above results, $\mathbf{T}$ was modified with a short PEG chain that consisted of four ethylene glycol units in the following experiments. Further, the molar ratio of the two monomers $\mathbf{T}$ and modified $\mathbf{T}$ was kept constant at 1:1.

The sensitivity to EtOH was also evaluated with the other four sensors in the array, which would be introduced in the following part, and the results are summarized in Figure S7. The four mixed sensors all showed higher sensitivity than sensor $\mathbf{T}$, proving the feasibility of improving the sensitivity to EtOH by introducing a second DA monomer into the vesicles. In addition, sensor T+T-PEG-SH showed a much higher sensitivity than sensor $\mathbf{T}+\mathbf{T}-\mathbf{S H}$, which validated our assumption that increasing hydrophilicity helped to improve the sensitivity of the sensor. In general, the five sensors showed quite different responses toward $\mathrm{EtOH}$ at low concentrations, which laid the foundation for using the sensor array to discriminate commercial beers.

Detection of 4-Vinyl Guaiacol (4-VG). 4-VG is a key beer flavoring compound. It can be found naturally in most beers (ales in particular), which endows the beer with a spicy, clove-like taste. However, at high concentrations, it presents as 
a medicinal, off-flavor note. ${ }^{51}$ Inspired by a previous report where imidazole-functionalized PDA sensors reacted with $\mathrm{CO}_{2}$ to form carbamate anions, which led to the color switch of the system, ${ }^{52}$ we selected the well-known thiol-ene click reaction between a thiol group and an alkene ${ }^{53}$ to design sensors for 4VG detection. Therefore, DA T-SH with a thiol group as the head group was synthesized from T. Sensor T+T-SH was assembled using a 1:1 molar ratio of the two DA monomers. It was anticipated that in the presence of UV light (radical addition), T-SH would react with the double bond on 4-VG via a radical promoted thiol-ene reaction (Supporting Information Scheme S4), which would lead to the disturbance of the PDA backbone and thus a color switch. However, no obvious color changes were observed when sensor T+T-SH was exposed to $10 \mathrm{mM} 4-\mathrm{VG}$ in the presence of the photoinitiator 2,2-dimethoxy-2-phenylacetophenone (DMAP) in water, even after incubation for $24 \mathrm{~h}$. This was likely due to the poor solubility of DMAP in water, which hindered the reaction.

Surprisingly, with the sensors in hand, when we tested the response of all the sensors assembled thus far toward 4-VG in the absence of DMAP, T+T-PEG1 and T+T-SH showed a relatively high sensitivity toward $4-\mathrm{VG}$, while $\mathrm{T}$ was almost nonsensitive. Especially, sensor T+T-PEG1 showed significant differences in CRs as the 4-VG concentration increased from 0.01 to $1 \mathrm{mM}$ (Supporting Information, Figure S8). A CR of $\sim 8 \%$ was observed for sensor T+T-PEG1 when the concentration of $4-\mathrm{VG}$ reached $1 \mathrm{mM}$. When considering a DA that carries both, the PEG chain and the thiol head group (T-PEG-SH) for the sensor T+T-PEG-SH assembly, an even higher CR ( $15.9 \%$ for $1 \mathrm{mM}$ of $4-\mathrm{VG})$ was observed (Figure $3 \mathrm{a})$, which again indicated the significance of hydrophilicity of the sensor. Quantitative analysis of the CRs suggested that

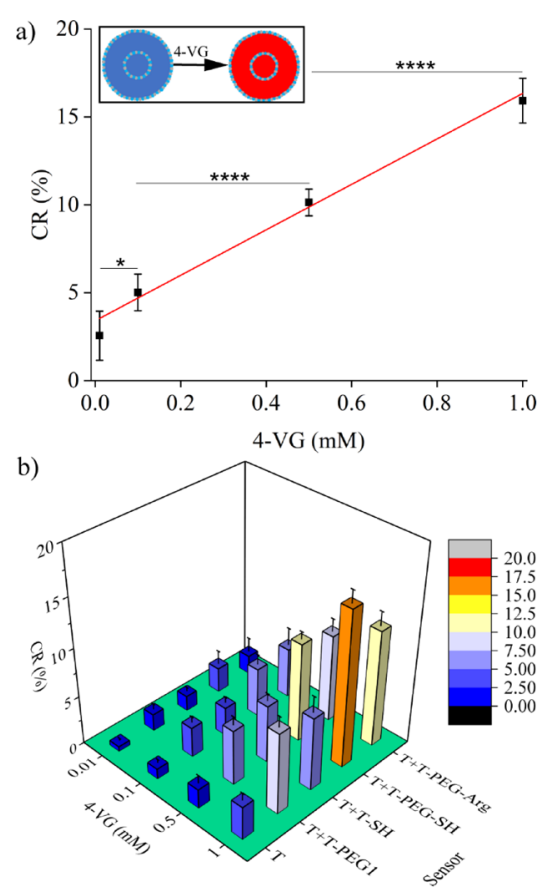

Figure 3. 4-VG detection: (a) Colorimetric responses of sensor $\mathbf{T}+\mathbf{T}$ PEG-SH toward increasing concentrations of 4-VG. The red line is the linear fitting curve. (b) Colorimetric responses of the five sensors toward different concentrations of $4-\mathrm{VG}$. The data are expressed as mean \pm SD. $(n=5, * p<0.1, * * * * p<0.0001)$. sensor T+T-PEG-SH exhibited a linear response to $4-\mathrm{VG}$ in water at the given concentrations $(0.01$ to $1 \mathrm{mM})$ with a good correlation $\left(R^{2}=0.989\right)$, which highlighted the feasibility of sensor T+T-PEG-SH to quantitatively detect 4-VG (Figure 3a). The detection limit was calculated to be $0.19 \mathrm{mM}(28.5$ ppm) in water.

Furthermore, all modified sensors used in this manuscript had higher sensitivity to 4-VG compared to sensor $\mathbf{T}$ (Figure 3b) with sensor T+T-PEG-SH outperforming the others (Supporting Information, Table S3). It is assumed that the sensitivity for 4-VG arose from the affinity between 4-VG and the head groups of the sensors. Such affinity-induced sensing is usually nonspecific and irreversible, which also explains why several sensors showed certain sensitivity to 4-VG.

Detection of Diacetyl. Diacetyl is generated in the early stage of the fermentation cycle as a byproduct of the synthesis of valine, responsible for the buttery flavor in beers. ${ }^{54}$ While diacetyl is desired for certain types of brews, it is often considered as a rancid off-flavor.

The hydrolysis reaction between diacetyl and arginine was considered to design a PDA sensor for the detection of diacetyl. It has been reported that diacetyl modifies arginine residues and two types of major products are generated, the cyclized forms and open-chain components (Figure 4a). ${ }^{55}$ Although the hydrolysis takes place rather slowly, we
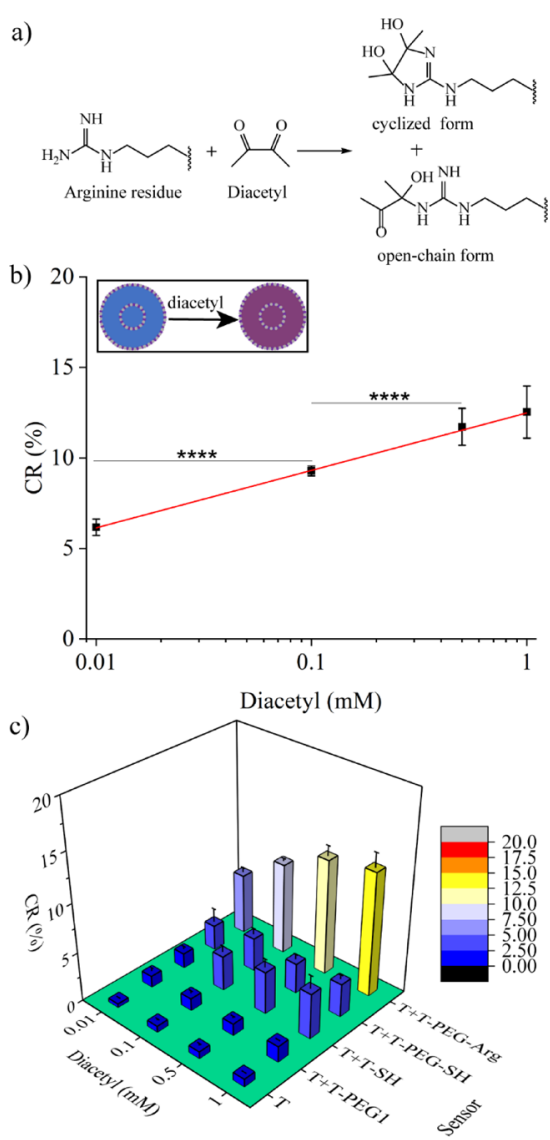

Figure 4. Diacetyl detection: (a) Hydrolysis of arginine in the presence of diacetyl. (b) Colorimetric responses of sensor T+T-PEGArg toward increasing concentrations of diacetyl. The red line is the linear fitting curve. (c) Colorimetric responses of the five sensors toward different concentrations of diacetyl. The data are expressed as mean \pm SD. $(n=5, * * * * p<0.0001)$. 
envisioned that even a small portion of hydrolysis on argininemodified PDAs would disturb the conjugated backbone, leading to a distinct color change. Therefore, two different DAs were synthesized. Arginine was directly attached to $T$ via a coupling reaction, and DA T-Arg was obtained. In addition, a PEG chain was introduced between $\mathbf{T}$ and arginine, and DA TPEG-Arg was synthesized. T-Arg could be polymerized at the solid state as the color clearly changed after UV irradiation for 2 min (Supporting Information, Figure S9). However, sensors made from $\mathbf{T}$ and $\mathbf{T}$-Arg led to precipitation after storage overnight at low temperatures, likely due to the poor water solubility of T-Arg. On the other hand, sensor T+T-PEG-Arg exhibited much better stability and high sensitivity toward diacetyl with a CR of $\sim 12.5 \%$ when exposed to $1 \mathrm{mM}$ diacetyl (Figure $4 \mathrm{~b})$. An excellent linear correlation was shown $\left(R^{2}=\right.$ 0.999) in the tested concentration range, and the detection limit for diacetyl was calculated to be $0.15 \mathrm{mM}$ (13.0 ppm). Further, all modified sensors used in this manuscript had higher sensitivity to diacetyl compared to sensor $\mathbf{T}$ (Figure 4c), but sensor T+T-PEG-Arg exhibited significantly higher CR compared to all of them (Supporting Information, Table S4). Unlike 4-VG, the sensitivity for diacetyl mainly stemmed from the reaction between diacetyl and arginine residues, which explains why only T+T-PEG-Arg showed a high CR while the other sensors did not.

Other Flavoring Compounds. Except for 4-VG and diacetyl, other ubiquitous flavoring compounds exist in beers, including ethyl acetate, isobutanol, 2-phenylethanol, 1propanol, and isopentanol. The sensitivity of the sensors toward these compounds was also determined. However, in general, lower CR was obtained for these flavoring compounds as compared to $4-\mathrm{VG}$ and diacetyl (Supporting Information, Figure S10).

Mimicking the Beer Environment. Considering that the sensing environment in beers is much more complex than the individual compounds in water, we aimed to determine whether the sensitivity of the sensors toward individual analytes would be affected by the presence of other analytes. The seven analytes tested above, including 4-VG, diacetyl, ethyl acetate, isobutanol, 2-phenylethanol, 1-propanol, and isopentanol were mixed, and the concentration of each analyte was kept at $1 \mathrm{mM}$. The experiments were done without (mixture A) and with (mixture B) the presence of EtOH (12 vol \%) to mimic an alcohol-free and an alcoholic beer environment. The responses of sensor T+T-PEG1 and T+TPEG-SH toward the mixtures were evaluated, and the results were compared to their responses to the individual compounds. The CR for the mixtures was approximately the added-up CR from the response to the individual compounds for both sensors (Figure 5). This was an exciting result, especially since it indicated that the CRs were not dominated by the presence of EtOH in the mixtures, which means in turn that the sensors could be potentially used to discriminate complex mixtures.

Beer Discrimination. Finally, the five PDA sensors were used to distinguish different commercial beers. First, we chose four beers with different volumetric amounts of $\mathrm{EtOH}$ ranging from 0.09 to $10 \mathrm{vol} \%$ : Tuborg Super Light, Brewmasters IPA, Tuborg Fine Festival, and Årgangsbryg (for more details, see Supporting Information Table S5 and Figure S11). The discriminating power was anticipated to arise from the different patterns generated based on the CRs of the sensors when exposed to the different beers.

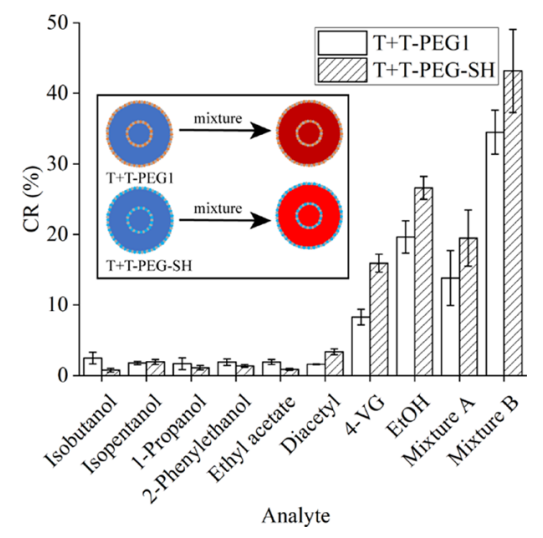

Figure 5. Colorimetric responses of sensor $\mathbf{T}+\mathbf{T}$-PEG1 and sensor $\mathbf{T}$ +T-PEG-SH toward different individual analytes and a mixture of all the analytes without (mixture A) and with (mixture B) EtOH (12 vol $\%)$. The data are expressed as mean \pm SD. $(n=5)$.

The sensor array consisting of five sensors generated a data set with $160 \mathrm{CR}$ observations for four beers $(5$ sensors $\times 4$ beers $\times 8$ repeats). A multivariate analysis method was applied to be able to use and interpret the patterns. Herein, principal component analysis (PCA) was chosen. The goal of PCA is to decrease the dimensionality of the dataset (number of features/variables) while keeping most of the original variability (information) by grouping similar observations together. Essentially, it takes the data points (changes in CR for each sensor in the array) for all analytes (different beers) and creates a set of orthogonal eigenvectors (principal components) to include the maximum variance. Therefore, through the construction of a set of principal components (PCs), which act as a new reduced set of variables, PCA concentrates the data's variation among analytes into the minimum number of dimensions. Hence, for a given data set, the first PC has the highest explanatory power once it describes as much of variability of the data as possible. ${ }^{56-58}$ In our case, PC1 and PC2 accounted for $61.2 \%$ of the data variance (Figure 6a). The PCA plot showed four groups, with clear separation of three out of the four beers: Årgangsbryg, Tuborg Fine Festival, and Tuborg Super Light. Årgangsbryg with the highest content of EtOH (10.0 vol \%) could be clearly distinguished from the other three, which was likely a result of the high sensitivity of the sensors toward EtOH. While Brewmasters IPA, which has an EtOH content between Tuborg Fine Festival and Tuborg Super Light, displayed a small overlap with both of them, which indicated that Brewmasters IPA could be generally distinguished from the other two using the sensor array, but some errors are expected. This analysis demonstrated the discriminating power of the sensor array to successfully differentiate beers with different EtOH contents. Further, to expand and corroborate the previous results obtained with PCA, the hierarchical clustering analysis method (HCA) was used. HCA is a statistical procedure widely used due to its user-friendly visual representation. It is an algorithm that groups similar observations into groups called clusters. The goal is to obtain a set of clusters that are distinct from any other cluster, but the observations within each cluster are similar to each other. There are several methods to define the clusters. In this work, Ward's method was chosen. (A more detailed explanation of the HCA method can be found in the Supporting Information.) The HCA dendrogram showed that the sensor 
a)

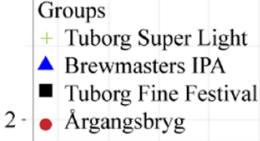

+ Tuborg Super Light

- Brewmasters IPA

- Tuborg Fine Festival

2. Årgangsbryg

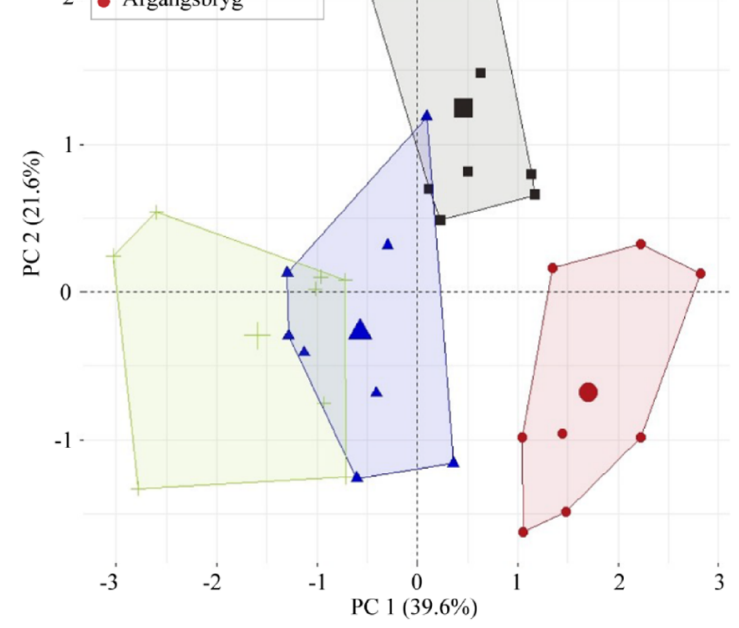

b)

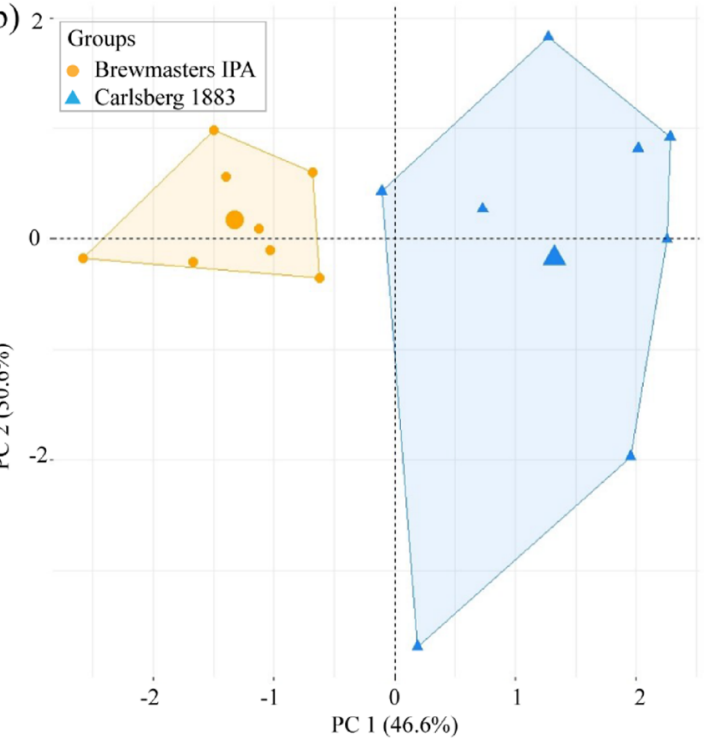

Figure 6. Beer discrimination. (a) PCA plot of the sensor array after exposure to four different commercial beers with different EtOH contents. (b) PCA plot of the sensor array after exposure to the two different commercial beers with similar EtOH contents.

array had a high potential for separation of the four beers, even though there are some misidentifications (Supporting Information, Figure S12).

As a follow-up, to further understand the discriminating power of the sensor array, we aimed to separate beers with similar EtOH contents. Six beers with $\mathrm{EtOH}$ contents ranging from 4.5 to 5.8 vol \% were selected: Tuborg Rå, Tuborg Classic, Carlsberg 1883, Brewmasters IPA, Guld Tuborg, and Carlsberg Sort Guld. When considering all six beers, the PCA analysis showed severe overlapping (Supporting Information, Figure S13). However, if being more selective with the chosen beer and focusing on only Brewmasters IPA and Carlsberg 1883, clear separation was found (Figure 6b), showing that the $\mathrm{EtOH}$ content was not always the sole discriminator.

\section{CONCLUSIONS}

Herein, we report the design, synthesis, and application of a solution-based sensor array derived from nanoscale PDA vesicles to discriminate commercial beers. The head groups and the compositions of the diacetylenes used for the selfassembly of the PDA sensors played a vital role in tailoring the ability of the sensors to detect different $\mathrm{EtOH}$ concentrations and different flavoring molecules and to distinguish different beers. Among the five sensors used to construct the sensor array, sensor T+T-PEG-SH showed the lowest detection limit (0.19 $\mathrm{mM})$ with the highest CR (15.9\%) for 4-VG, while sensor T+T-PEG-Arg showed the lowest detection limit (0.15 $\mathrm{mM}$ ) with the highest CR (12.5\%) for diacetyl. Although the detection limits for these two compounds were higher than their respective thresholds in commercial beers, ${ }^{51,54}$ discriminating beers was possible. This latter aspect was most successful when the EtOH content varied. However, selected beers of similar EtOH contents could be separated with our current array. We would like to note that quantification of the beer compositions as it is possible with GC-MS could not be accomplished with our current sensor array. This was never the aim since we targeted the separation of entire flavor profiles. Taken together, this approach represents a first effort for the fabrication of low-cost sensors for fast discrimination of complex mixtures focusing on the beer industry.

\section{ASSOCIATED CONTENT}

\section{SI Supporting Information}

The Supporting Information is available free of charge at https://pubs.acs.org/doi/10.1021/acsanm.0c00187.

Synthetic procedures of the monomers, DLS data, UVvis spectra, photos, detailed information of the tested beers, HCA analysis, and PCA plot (PDF)

\section{AUTHOR INFORMATION}

\section{Corresponding Author}

Brigitte Städler - Interdisciplinary Nanoscience Center (iNANO), Aarhus University, 8000 Aarhus, Denmark; 다이.org/0000-0002-7335-3945; Email: bstadler@ inano.au.dk

\section{Authors}

Xiaomin Qian - Interdisciplinary Nanoscience Center (iNANO), Aarhus University, 8000 Aarhus, Denmark

Carina L. Gargalo - Process and Systems Engineering Center (PROSYS), Department of Chemical and Biochemical Engineering, Technical University of Denmark, 2800 Kongens Lyngby, Denmark; 이이.org/0000-0002-8740-9591

Krist V. Gernaey - Process and Systems Engineering Center (PROSYS), Department of Chemical and Biochemical Engineering, Technical University of Denmark, 2800 Kongens Lyngby, Denmark

Complete contact information is available at: https://pubs.acs.org/10.1021/acsanm.0c00187

\section{Notes}

The authors declare no competing financial interest.

\section{ACKNOWLEDGMENTS}

This work was supported by the Innovation Fund Denmark (grant no. 7045-00014A). 


\section{REFERENCES}

(1) Heberle, I.; Liebminger, A.; Weimar, U.; Göpel, W. Optimised sensor arrays with chromatographic preseparation: characterisation of alcoholic beverages. Sens. Actuators B Chem. 2000, 68, 53-57.

(2) Medina, S.; Pereira, J. A.; Silva, P.; Perestrelo, R.; Câmara, J. S. Food fingerprints - A valuable tool to monitor food authenticity and safety. Food Chem. 2019, 278, 144-162.

(3) Latha, R. S.; Lakshmi, P. K. Electronic tongue: An analytical gustatory tool. J. Adv. Pharm. Technol. Res. 2012, 3, 3-8.

(4) Qian, X.; Städler, B. Recent Developments in PolydiacetyleneBased Sensors. Chem. Mater. 2019, 31, 1196-1222.

(5) Jelinek, R.; Ritenberg, M. Polydiacetylenes - recent molecular advances and applications. RSC Adv. 2013, 3, 21192-21201.

(6) Wen, J. T.; Roper, J. M.; Tsutsui, H. Polydiacetylene Supramolecules: Synthesis, Characterization, and Emerging Applications. Ind. Eng. Chem. Res. 2018, 57, 9037-9053.

(7) Huo, J.; Deng, Q.; Fan, T.; He, G.; Hu, X.; Hong, X.; Chen, H.; Luo, S.; Wang, Z.; Chen, D. Advances in polydiacetylene development for the design of side chain groups in smart material applications - a mini review. Polym. Chem. 2017, 8, 7438-7445.

(8) Wegner, G. Topochemische Reaktionen von Monomeren mit konjug ierten Dreifachbindungen. Z. Naturforsch. B 1969, 24, 824832.

(9) Park, I. S.; Park, H. J.; Kim, J. M. A soluble, low-temperature thermochromic and chemically reactive polydiacetylene. ACS Appl. Mater. Interfaces 2013, 5, 8805-8812.

(10) Huo, J.; Hu, Z.; He, G.; Hong, X.; Yang, Z.; Luo, S.; Ye, X.; Li, Y.; Zhang, Y.; Zhang, M.; Chen, H.; Fan, T.; Zhang, Y.; Xiong, B.; Wang, Z.; Zhu, Z.; Chen, D. High temperature thermochromic polydiacetylenes: Design and colorimetric properties. Appl. Surf. Sci. 2017, 423, 951-956.

(11) Dei, S.; Matsumoto, A.; Matsumoto, A. Thermochromism of Polydiacetylenes in the Solid State and in Solution by the SelfOrganization of Polymer Chains Containing No Polar Group. Macromolecules 2008, 41, 2467-2473.

(12) Ampornpun, S.; Montha, S.; Tumcharern, G.; Vchirawongkwin, V.; Sukwattanasinitt, M.; Wacharasindhu, S. Odd-Even and Hydrophobicity Effects of Diacetylene Alkyl Chains on Thermochromic Reversibility of Symmetrical and Unsymmetrical Diyndiamide Polydiacetylenes. Macromolecules 2012, 45, 9038-9045.

(13) Lee, S.; Lee, J.; Lee, M.; Cho, Y. K.; Baek, J.; Kim, J.; Park, S.; Kim, M. H.; Chang, R.; Yoon, J. Construction and Molecular Understanding of an Unprecedented, Reversibly Thermochromic BisPolydiacetylene. Adv. Funct. Mater. 2014, 24, 3699-3705.

(14) Park, I. S.; Park, H. J.; Jeong, W.; Nam, J.; Kang, Y.; Shin, K.; Chung, H.; Kim, J.-M. Low Temperature Thermochromic Polydiacetylenes: Design, Colorimetric Properties, and Nanofiber Formation. Macromolecules 2016, 49, 1270-1278.

(15) Shin, G.; Khazi, M. I.; Kundapur, U.; Kim, B.; Kim, Y.; Lee, C. W.; Kim, J.-M. Cation-Directed Self-Assembly of Macrocyclic Diacetylene for Developing Chromogenic Polydiacetylene. ACS Macro Lett. 2019, 610-615.

(16) Cheng, Q.; Stevens, R. C. Charge-Induced Chromatic Transition of Amino Acid-Derivatized Polydiacetylene Liposomes. Langmuir 1998, 14, 1974-1976.

(17) Song, J.; Cheng, Q.; Kopta, S.; Stevens, R. C. Modulating artificial membrane morphology: $\mathrm{pH}$-induced chromatic transition and nanostructural transformation of a bolaamphiphilic conjugated polymer from blue helical ribbons to red nanofibers. J. Am. Chem. Soc. 2001, 123, 3205-3213.

(18) Chen, X.; Hong, L.; You, X.; Wang, Y.; Zou, G.; Su, W.; Zhang, Q. Photo-controlled molecular recognition of alpha-cyclodextrin with azobenzene containing polydiacetylene vesicles. Chem. Commun. 2009, 1356-1358.

(19) Nallicheri, R. A.; Rubner, M. F. Investigations of the mechanochromic behavior of poly(urethane-diacetylene) segmented copolymers. Macromolecules 1991, 24, 517-525.
(20) Terada, H.; Imai, H.; Oaki, Y. Visualization and Quantitative Detection of Friction Force by Self-Organized Organic Layered Composites. Adv. Mater. 2018, 30, 1801121.

(21) Ma, Z.; Li, J.; Liu, M.; Cao, J.; Zou, Z.; Tu, J.; Jiang, L. Colorimetric Detection of Escherichia coli by Polydiacetylene Vesicles Functionalized with Glycolipid. J. Am. Chem. Soc. 1998, 120, 1267812679 .

(22) Park, C. H.; Kim, J. P.; Lee, S. W.; Jeon, N. L.; Yoo, P. J.; Sim, S. J. A Direct, Multiplex Biosensor Platform for Pathogen Detection Based on Cross-linked Polydiacetylene (PDA) Supramolecules. Adv. Funct. Mater. 2009, 19, 3703-3710.

(23) Zhou, G.; Wang, F.; Wang, H.; Kambam, S.; Chen, X. Colorimetric and fluorometric detection of neomycin based on conjugated polydiacetylene supramolecules. Macromol. Rapid Commun. 2013, 34, 944-948.

(24) Kang, D. H.; Jung, H. S.; Ahn, N.; Lee, J.; Seo, S.; Suh, K. Y.; Kim, J.; Kim, K. Biomimetic detection of aminoglycosidic antibiotics using polydiacetylene-phospholipids supramolecules. Chem. Commun. 2012, 48, 5313-5315.

(25) Ahn, D. J.; Chae, E. H.; Lee, G. S.; Shim, H. Y.; Chang, T. E.; Ahn, K. D.; Kim, J. M. Colorimetric reversibility of polydiacetylene supramolecules having enhanced hydrogen-bonding under thermal and pH stimuli. J. Am. Chem. Soc. 2003, 125, 8976-8977.

(26) Alekseev, A.; Ihalainen, P.; Ivanov, A.; Domnin, I.; Rosqvist, E.; Lemmetyinen, H.; Vuorimaa-Laukkanen, E.; Peltonen, J.; Vyaz'min, S. Stable blue phase polymeric Langmuir-Schaefer films based on unsymmetrical hydroxyalkadiynyl $\mathrm{N}$-arylcarbamate derivatives. Thin Solid Films 2018, 645, 108-118.

(27) Wang, Y.; Pei, H.; Jia, Y.; Liu, J.; Li, Z.; Ai, K.; Lu, Z.; Lu, L. Synergistic Tailoring of Electrostatic and Hydrophobic Interactions for Rapid and Specific Recognition of Lysophosphatidic Acid, an Early-Stage Ovarian Cancer Biomarker. J. Am. Chem. Soc. 2017, 139, $11616-11621$.

(28) Li, S.; Zhang, L.; Jiang, J.; Meng, Y.; Liu, M. Self-Assembled Polydiacetylene Vesicle and Helix with Chiral Interface for Visualized Enantioselective Recognition of Sulfinamide. ACS Appl. Mater. Interfaces 2017, 9, 37386-37394.

(29) Wang, D. E.; Yan, J.; Jiang, J.; Liu, X.; Tian, C.; Xu, J.; Yuan, M. S.; Han, X.; Wang, J. Polydiacetylene liposomes with phenylboronic acid tags: a fluorescence turn-on sensor for sialic acid detection and cell-surface glycan imaging. Nanoscale 2018, 10, 4570-4578.

(30) Chae, S.; Lee, J. P.; Kim, J.-M. Mechanically Drawable Thermochromic and Mechanothermochromic Polydiacetylene Sensors. Adv. Funct. Mater. 2016, 26, 1769-1776.

(31) Kang, D. H.; Jung, H. S.; Ahn, N.; Yang, S. M.; Seo, S.; Suh, K. Y.; Chang, P. S.; Jeon, N. L.; Kim, J.; Kim, K. Janus-compartmental alginate microbeads having polydiacetylene liposomes and magnetic nanoparticles for visual lead(II) detection. ACS Appl. Mater. Interfaces 2014, 6, 10631-10637.

(32) Wang, T.; Guo, Y.; Wan, P.; Sun, X.; Zhang, H.; Yu, Z.; Chen, $\mathrm{X}$. A flexible transparent colorimetric wrist strap sensor. Nanoscale 2017, 9, 869-874.

(33) Lee, S.; Lee, K. M.; Lee, M.; Yoon, J. Polydiacetylenes bearing boronic acid groups as colorimetric and fluorescence sensors for cationic surfactants. ACS Appl. Mater. Interfaces 2013, 5, 4521-4526.

(34) Wang, D. E.; Zhao, L.; Yuan, M. S.; Chen, S. W.; Li, T.; Wang, J. Fabrication of Polydiacetylene Liposome Chemosensor with Enhanced Fluorescent Self-Amplification and Its Application for Selective Detection of Cationic Surfactants. ACS Appl. Mater. Interfaces 2016, 28231-28240.

(35) Park, J.; Ku, S. K.; Seo, D.; Hur, K.; Jeon, H.; Shvartsman, D.; Seok, H. K.; Mooney, D. J.; Lee, K. Label-free bacterial detection using polydiacetylene liposomes. Chem. Commun. 2016, 52, 1034610349.

(36) Lee, S.; Cheng, H.; Chi, M.; Xu, Q.; Chen, X.; Eom, C. Y.; James, T. D.; Park, S.; Yoon, J. Sensing and antibacterial activity of imidazolium-based conjugated polydiacetylenes. Biosens. Bioelectron. 2016, 77, 1016-1019. 
(37) Wang, M.; Wang, F.; Wang, Y.; Zhang, W.; Chen, X. Polydiacetylene-based sensor for highly sensitive and selective $\mathrm{Pb}^{2+}$ detection. Dyes Pigm. 2015, 120, 307-313.

(38) Zhang, Y.; Northcutt, J.; Hanks, T.; Miller, I.; Pennington, B.; Jelinek, R.; Han, I.; Dawson, P. Polydiacetylene sensor interaction with food sanitizers and surfactants. Food Chem. 2017, 221, 515-520.

(39) Silbert, L.; Ben Shlush, I.; Israel, E.; Porgador, A.; Kolusheva, S.; Jelinek, R. Rapid Chromatic Detection of Bacteria by Use of a New Biomimetic Polymer Sensor. Appl. Environ. Microbiol. 2006, 72, 7339-7344.

(40) Park, M.-K.; Kim, K.-W.; Ahn, D. J.; Oh, M.-K. Label-free detection of bacterial RNA using polydiacetylene-based biochip. Biosens. Bioelectron. 2012, 35, 44-49.

(41) Wu, J.; Zawistowski, A.; Ehrmann, M.; Yi, T.; Schmuck, C. Peptide Functionalized Polydiacetylene Liposomes Act as a Fluorescent Turn-On Sensor for Bacterial Lipopolysaccharide. J. Am. Chem. Soc. 2011, 133, 9720-9723.

(42) Okada, S.; Peng, S.; Spevak, W.; Charych, D. Color and Chromism of Polydiacetylene Vesicles. Acc. Chem. Res. 1998, 31, 229-239.

(43) Alami, M.; Ferri, F. A convenient route to unsymmetrical conjugated diynes. Tetrahedron Lett. 1996, 37, 2763-2766.

(44) Charoenthai, N.; Pattanatornchai, T.; Wacharasindhu, S.; Sukwattanasinitt, M.; Traiphol, R. Roles of head group architecture and side chain length on colorimetric response of polydiacetylene vesicles to temperature, ethanol and $\mathrm{pH}$. J. Colloid Interface Sci. 2011, $360,565-573$.

(45) Reichert, A.; Nagy, J. O.; Spevak, W.; Charych, D. Polydiacetylene Liposomes Functionalized with Sialic Acid Bind and Colorimetrically Detect Influenza Virus. J. Am. Chem. Soc. 1995, 117, 829-830.

(46) Lee, J.; Chang, H. T.; An, H.; Ahn, S.; Shim, J.; Kim, J. M. A protective layer approach to solvatochromic sensors. Nat. Commun. 2013, 4, 2461.

(47) Tu, M.-C.; Cheema, J. A.; Yildiz, U. H.; Palaniappan, A.; Liedberg, B. Vapor phase solvatochromic responses of polydiacetylene embedded matrix polymers. J. Mater. Chem. C 2017, 5, 1803-1809.

(48) Park, D. H.; Heo, J. M.; Jeong, W.; Yoo, Y. H.; Park, B. J.; Kim, J. M. Smartphone-Based VOC Sensor Using Colorimetric Polydiacetylenes. ACS Appl. Mater. Interfaces 2018, 10, 5014-5021.

(49) Kang, D. H.; Jung, H. S.; Kim, K.; Kim, J. Mussel-Inspired Universal Bioconjugation of Polydiacetylene Liposome for DropletArray Biosensors. ACS Appl. Mater. Interfaces 2017, 9, 42210-42216.

(50) Li, Y.; Wang, L.; Yin, X.; Ding, B.; Sun, G.; Ke, T.; Chen, J.; Yu, J. Colorimetric strips for visual lead ion recognition utilizing polydiacetylene embedded nanofibers. J. Mater. Chem. A 2014, 2, 18304-18312.

(51) McMurrough, I.; Madigan, D.; Donnelly, D.; Hurley, J.; Doyle, A.-M.; Hennigan, G.; McNulty, N.; Smyth, M. R. Control of Ferulic Acid and 4-Vinyl Guaiacol in Brewing. J. Inst. Brew. 1996, 102, 327332.

(52) Xu, Q.; Lee, S.; Cho, Y.; Kim, M. H.; Bouffard, J.; Yoon, J. Polydiacetylene-based colorimetric and fluorescent chemosensor for the detection of carbon dioxide. J. Am. Chem. Soc. 2013, 135, 1775117754.

(53) Hoyle, C. E.; Bowman, C. N. Thiol-ene click chemistry. Angew. Chem., Int. Ed. Engl. 2010, 49, 1540-1573.

(54) Krogerus, K.; Gibson, B. R. 125th Anniversary Review: Diacetyl and its control during brewery fermentation. J. Inst. Brew. 2013, 119, 86-97.

(55) Mathews, J. M.; Watson, S. L.; Snyder, R. W.; Burgess, J. P.; Morgan, D. L. Reaction of the butter flavorant diacetyl (2,3butanedione) with $\mathrm{N}$-alpha-acetylarginine: a model for epitope formation with pulmonary proteins in the etiology of obliterative bronchiolitis. J. Agric. Food Chem. 2010, 58, 12761-12768.

(56) Zhang, C.; Bailey, D. P.; Suslick, K. S. Colorimetric sensor arrays for the analysis of beers: a feasibility study. J. Agric. Food Chem. 2006, 54, 4925-4931.
(57) Ghasemi-Varnamkhasti, M.; Rodríguez-Méndez, M. L.; Mohtasebi, S. S.; Apetrei, C.; Lozano, J.; Ahmadi, H.; Razavi, S. H.; Antonio de Saja, J. Monitoring the aging of beers using a bioelectronic tongue. Food Control 2012, 25, 216-224.

(58) Smyth, H.; Cozzolino, D. Instrumental methods (spectroscopy, electronic nose, and tongue) as tools to predict taste and aroma in beverages: advantages and limitations. Chem. Rev. 2013, 113, 14291440. 